\title{
Cetoacidose Diabética em Adultos - Atualização de uma Complicação Antiga
}

\section{revisão}

\author{
BIANCA BARONE \\ MELANIE RODACKI \\ maria Claudia Peixoto Cenci \\ LENITA ZAJDENVERG \\ ADOLPHO MILECH \\ JOSÉ EGIDIO P. DE OLIVEIRA
}

Instituto Estadual de Diabetes e Endocrinologia Luiz Capriglione - IEDE (BB \& MR) e Serviço de Nutrologia do Hospital Universitário Clementino Fraga Filho - HUCFF (BB, MR, MCPC,

LZ, AM \& JEPO), Universidade Federal do Rio de Janeiro (UFRJ), Rio de Janeiro, RJ.

Recebido em 09/03/07 Aceito em 22/06/07
RESUMO

A cetoacidose diabética é uma complicação aguda do Diabetes Mellitus (DM) caracterizada por hiperglicemia, acidose metabólica, desidratação e cetose, na vigência de deficiência profunda de insulina. Acomete principalmente pacientes com DM tipo 1 e geralmente é precipitada por condições infecciosas, uso inadequado de insulina ou desconhecimento do diagnóstico de diabetes. Os autores revisam mecanismos fisiopatológicos, critérios diagnósticos e opções terapêuticas do distúrbio em adultos, bem como suas possíveis complicações. (Arq Bras Endocrinol Metab 2007;51/9:1434-1447)

Descritores: Cetoacidose diabética; Diabetes mellitus tipo 1; Tratamento; Fisiopatologia

\section{ABSTRACT}

Diabetic Ketoacidosis in Adults - Update of an Old Complication. Diabetic ketoacidosis is an acute complication of Diabetes Mellitus characterized by hyperglycemia, metabolic acidosis, dehydration, and ketosis, in patients with profound insulin deficiency. It occurs predominantly in patients with type 1 diabetes and is frequently precipitated by infections, insulin withdrawal or undiagnosed type 1 diabetes. The authors review its pathophysiology, diagnostic criteria and treatment options in adults, as well as its complications. (Arq Bras Endocrinol Metab 2007;51/9:1434-1447)

Keywords: Diabetic ketoacidosis; Type 1 diabetes; Treatment; Pathophysiology

A CETOACIDOSE Diabética (CAD) é uma complicação aguda do Diabetes Mellitus (DM) caracterizada por hiperglicemia, acidose metabólica, desidratação e cetose, na vigência de deficiência profunda de insulina. Acomete principalmente pacientes com DM tipo l (DMl) e geralmente é precipitada por condições infecciosas ou omissão da administração de insulina. Por vezes, a CAD pode ser a forma de apresentação clínica inicial do DMl ou mesmo do tipo 2 (DM2). A CAD é observada ao diagnóstico de DMl em 3 a 40\% dos pacientes diagnosticados com DMl (1-4). Recentemente, identificamos que, em nossa população, essa freqüência é de $32,8 \%$ (5).

Estima-se que a CAD tenha uma incidência anual de cerca de 1 a $5 \%$ da população, sem alterações significativas nos últimos anos (2). Existe uma incidência de CAD discretamente maior no sexo feminino, principalmente entre os adolescentes (6). No Brasil, existem poucos dados nacionais de incidência dessa complicação aguda (7-9).

Se diagnosticada e tratada de forma rápida e correta, a CAD tem bom prognóstico, sendo prontamente revertida na maioria das vezes (10). A mor- 
talidade por CAD teve uma queda significativa ao longo dos anos, chegando atualmente a menos de $5 \%$ dos casos em centros especializados (11-14). A mortalidade é maior nos extremos etários, podendo chegar a até $50 \%$ em pacientes idosos, com idade superior a 80 anos (15).

\section{FATORES PRECIPITANTES}

Na maioria das vezes, a CAD é precipitada por fatores desencadeantes identificáveis, dos quais os mais comuns são as infecções (30-39\%) e a omissão do uso de insulina $(21-49 \%)$.

Apesar de existirem inúmeros fatores já conhecidos associados ao desenvolvimento de CAD, algumas vezes essa complicação aguda ocorre sem que nenhum evento precipitante consiga ser evidenciado. A CAD pode, ainda, ser a forma inicial de apresentação do DM, tanto tipo 1 quanto tipo 2 (10,16-18). Há relatos, em diversas populações, especialmente de etnia hispânica ou africana, de indivíduos que apresentam CAD como forma de apresentação inicial do DM, porém que subseqüentemente desenvolvem curso clínico compatível com DM2, muitas vezes sendo possível a interrupção da insulinoterapia. Nesse subgrupo de pacientes, não são identificados auto-anticorpos associados ao DMl e freqüentemente há preservação ou recuperação da função pancreática endócrina. Essa subclasse peculiar de DM tem sido denominada de DM2 propenso a cetose ou diabetes flatbush.

Os principais fatores precipitantes da CAD são:

- Omissão da insulinoterapia

- Infecções (principalmente pulmonares)

- Situações de stress agudo: Acidente vascular encefálico (AVE), infarto agudo do miocárdio (IAM), pancreatite aguda, traumatismo, choque, hipovolemia, queimaduras, embolismo pulmonar, isquemia mesentérica etc.

- Gestação

- Outras patologias associadas (acromegalia, hemocromatose, hipertireoidismo)

- Problemas na bomba de insulina

- Abuso de substâncias (álcool, cocaína)

- Uso de medicamentos: corticosteróides, diuréticos (tiazídicos, clortalidona), agentes simpaticomiméticos (albuterol, dopamina, dobutamina, terbutalina, ritodrina), bloqueadores $\alpha$-adrenérgicos, bloqueadores $\beta$ adrenérgicos, pentamidina, inibidores de protease, somatostatina, fenitoína, antipsicóticos atípicos (loxapina, glucagon, interferon, bloqueador de canal de cálcio, clorpromazina, diazóxido, cimetidina, encainida, ácido etacrínico)
- Transtornos alimentares (compulsão alimentar, bulimia)

Nos pacientes em uso de sistemas de bomba de infusão subcutânea de insulina (BISCI), também chamados de "bomba de insulina", os fatores estão associados ao desenvolvimento de CAD por problemas intrínsecos à bomba (obstrução ou perda do posicionamento correto da cânula de infusão, presença de bolhas ou dobras no circuito de infusão, término de insulina contida no dispositivo, presença de infecção no local da cânula ou bateria fraca) ou por problemas extrínsecos ao sistema (baixa adesão do paciente às orientações recebidas, permanecendo desconectado do sistema por tempo superior àquele recomendado, sem administração compensatória de insulina por via convencional). Qualquer desses problemas promove a interrupção temporária da infusão de insulina e, como o depósito subcutâneo de insulina nesses pacientes é muito pequeno, a CAD pode se instalar em pouco tempo. Contudo, a maioria dos estudos tem demonstrado que o uso adequado da bomba de insulina, em pacientes corretamente selecionados, implica em um baixo risco de desenvolvimento de CAD, em comparação com o tratamento com múltiplas injeções de insulina (19-23). Contudo, isso ainda é controverso (24-26).

\section{FISIOPATOLOGIA}

A CAD resulta da deficiência profunda de insulina, seja ela absoluta ou relativa, e do excesso de hormônios contra-reguladores, como glucagon, cortisol e catecolaminas (27). Nesta circunstância, tecidos sensíveis à insulina passam a metabolizar principalmente gorduras ao invés de carboidratos. Como a insulina é um hormônio anabólico, sua deficiência favorece processos catabólicos, como lipólise, proteólise e glicogenólise. A lipólise resulta em liberação de ácidos graxos livres (AGL), que são oxidados no sistema microssomal hepático. O processo de oxidação de AGL é favorecido não só pelo excesso de substrato, como também por estímulo às vias metabólicas que oxidam ácidos graxos. A insulinopenia e o excesso de glucagon são capazes de ativar de forma indireta o sistema enzimático carnitina-acil-transferase, responsável pelo carreamento de AGL presentes no citossol dos hepatócitos para o sistema microssomal, onde serão oxidados. Isso ocorre, ao menos em parte, através da inibição da enzima acetil-CoA-carboxilase, que leva a uma redução da concentração plasmática de malonil-CoA, um intermediário da lipogênese que atua como inibidor da atividade desse sistema enzimático. 
Através da oxidação, os ácidos graxos são convertidos em acetil-CoA. Quando a produção de acetilCoA ultrapassa a capacidade de utilização hepática, a substância passa a ser convertida em corpos cetônicos (CC): acetoacetato, beta-hidroxibutirato $(\mathrm{BHB}$, que corresponde ao acetoacetato reduzido e é o principal CC da CAD) e acetona. Normalmente a relação entre BHB e acetoacetato é de $1: 1$ a 3:1. Na CAD, essa relação pode chegar a 10:1, pois a oxidação de ácidos graxos provoca redução do NAD em $\mathrm{NADH}^{+}$e a regeneração do NAD depende da redução do acetoacetato com conseqüente formação BHB. Acetona está presente em concentrações bem menores. A retenção dos CC no plasma provoca acidose metabólica com anion gap elevado $(28,29)$.

A hiperglicemia presente na CAD é causada por diminuição da utilização periférica de insulina, aumento da secreção hepática de glicose e diminuição de sua excreção. A secreção hepática exacerbada de glicose ocorre tanto por aumento da gliconeogênese (utilizando como principal substrato aminoácidos liberados na circulação sistêmica em decorrência da proteólise excessiva) quanto da glicogenólise $(16,30)$. A conseqüência é o aumento da osmolaridade plasmática, o que leva a um deslocamento de fluidos do espaço intra para o extracelular, com desenvolvimento de desidratação celular. Além disso, ultrapassando o limiar renal, surgem a glicosúria e a diurese osmótica, induzindo também perda de volume extracelular. Essa diurese osmótica é a principal causa de perda de fluidos na CAD $(31,32)$, embora outros fatores como vômito e hiperventilação também estejam envolvidos. Estima-se que haja perda de aproximadamente 5 a 10\% do peso corporal durante um episódio de CAD (33). A figura 1 esquematiza a fisiopatologia da CAD.

\section{QUADRO CLÍNICo}

No período antecedendo a $\mathrm{CAD}$, há manifestações referentes à descompensação metabólica, como poliúria, polifagia, polidipsia e cansaço. Com a instalação da $\mathrm{CAD}$, são observados anorexia, náuseas e vômitos, que podem agravar a desidratação. Cefaléia, mal-estar, parestesia e dor abdominal também são comuns (27). Com progressão da $\mathrm{CAD}$, pode haver alteração do nível de consciência, embora coma só ocorra em cerca de 10\% dos pacientes (10). É importante lembrar que a existência de DM nem sempre é mencionada, pois a CAD pode ser a forma de apresentação inicial da doença.

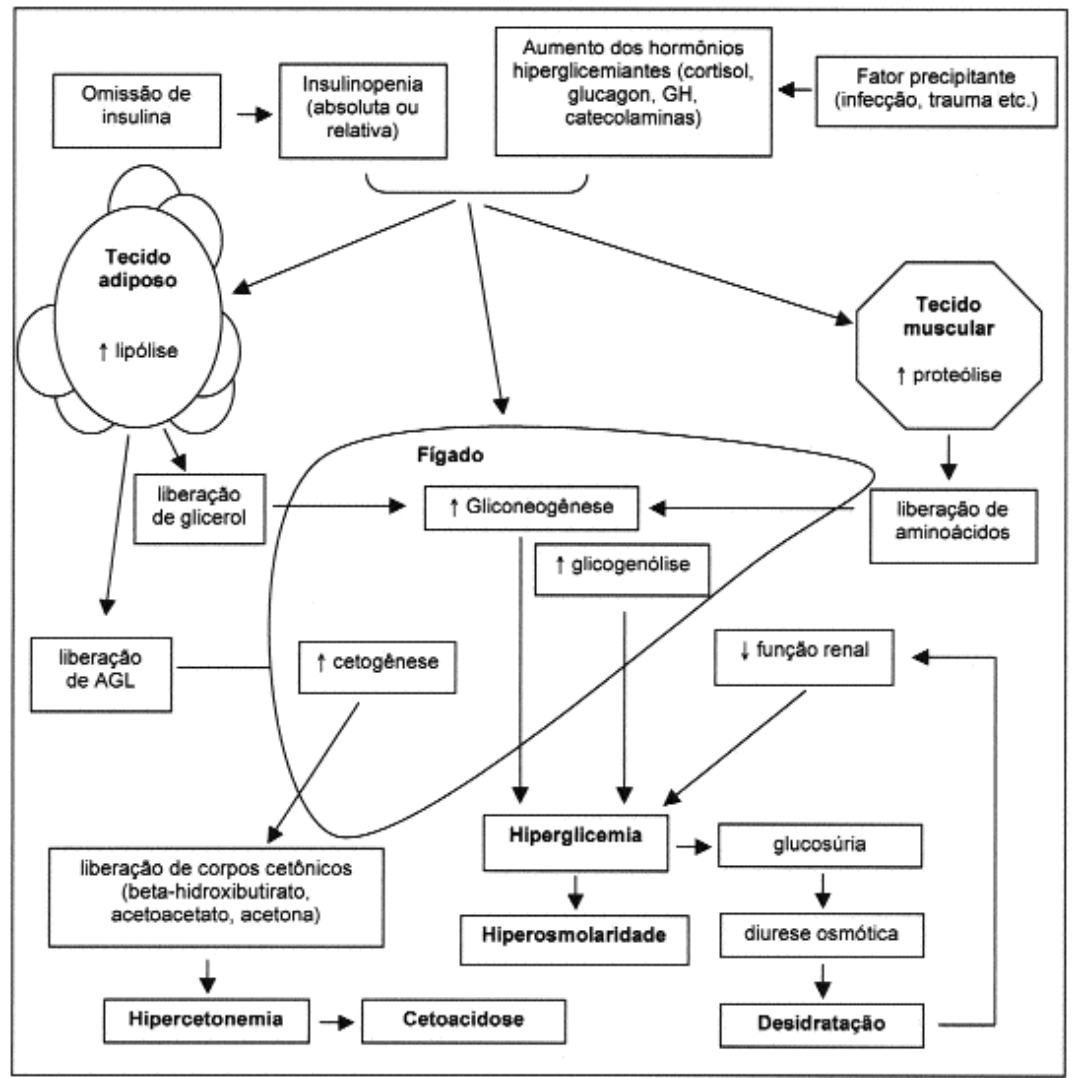

Figura 1. Patogênese da CAD. 
O exame físico revela desidratação, com mucosas ressecadas, turgor cutâneo diminuído e língua pregueada. Há taquicardia, hálito cetônico (de "maçã passada") e alterações do ritmo respiratório. Inicialmente há taquipnéia, que é seguida por ritmo de Kussmaul, podendo evoluir para respiração superficial em casos mais graves. Pode ainda haver hipotensão arterial. Na avaliação clínica, devem ser pesquisados sinais e sintomas sugestivos de possíveis condições desencadeantes, para que estas possam ser corrigidas, facilitando a recuperação da CAD (34). Alguns fatores são considerados como sugestivos de pior prognóstico, como a ocorrência da complicação em pacientes nos extremos etários, a presença de hipotensão arterial (27) ou de hipotermia (35).

\section{INVESTIGAÇÃO COMPLEMENTAR}

O diagnóstico definitivo de CAD consiste em demonstrar a presença de hiperglicemia (acima de $250 \mathrm{mg} / \mathrm{dl}$ ), acidose metabólica $(\mathrm{pH}<7,2$ e bicarbonato $<15$ $\mathrm{mEq} / \mathrm{L}$ ) e cetonemia (ou cetonúria) (10). A figura 2 resume o fluxograma para o diagnóstico da CAD e os demais exames complementares a serem solicitados.

\section{Glicemia}

É elevada, geralmente entre 250 e $850 \mathrm{mg} / \mathrm{dl}$, com a maioria dos casos entre 400 e $800 \mathrm{mg} / \mathrm{dl}$. Os menores valores são encontrados em crianças, pacientes em jejum prolongado, em uso de biguanidas ou no período gestacional. Na gestação, apesar de haver maiores níveis de hormônios hiperglicemiantes (cortisol, lactogênio placentário, progesterona), há aumento do consumo de glicose pelo feto e pela placenta, o que faz com que os níveis séricos de glicose diminuam rapidamente nos estados catabólicos (36). Os maiores valores de glicemia são vistos em pacientes idosos.

\section{Cetonemia}

Encontra-se elevada na CAD. Até recentemente, sua avaliação era realizada exclusivamente através da reação com o nitroprussiato de sódio, na qual o $\mathrm{BHB}$ não era dosado. Como este é o principal CC durante a CAD, obtinha-se resultados pouco fidedignos. Atualmente, existem métodos para dosagem de $\mathrm{BHB}$, inclusive com fitas reagentes usando sangue de modo similar à glicemia capilar, facilitando assim a sua realização (3739). Infelizmente, essas técnicas nem sempre são disponíveis em nosso meio. A dosagem de BHB através

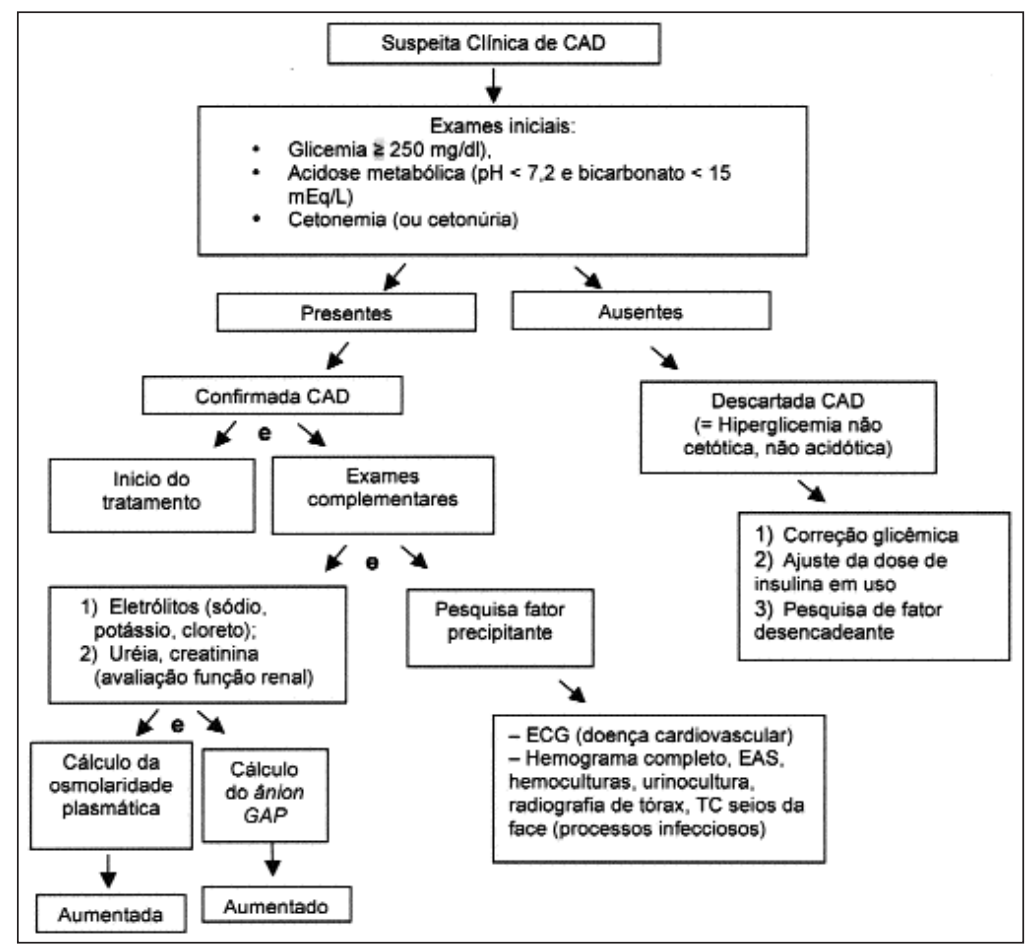

Adaptado de English \& Williams, 2004 (2) e de Kitabchi e cols., 2001 (62).

Figura 2. Fluxograma para o diagnóstico de CAD. 
desses testes pode ser muito útil para confirmação diagnóstica nos casos em que houver glicemia normal, como em idosos e gestantes (39). Estudos mais recentes têm sugerido que a dosagem capilar de BHB possa também ser extremamente útil para o diagnóstico domiciliar de CAD incipiente, facilitando a recuperação precoce. É recomendada a medida domiciliar de cetonemia no sangue capilar ou cetonúria com fita reagente própria para pacientes com DMl com hiperglicemia persistente acentuada $(\geq 300 \mathrm{mg} / \mathrm{dL}$ ou até menos, a critério clínico), especialmente na vigência de stress ou quadros infecciosos (40-44).

\section{Cetonúria}

É elevada e pode ser demonstrada no EAS. Permite diferenciação de $\mathrm{CAD}$ e outras causas de hiperglicemia associadas à acidose metabólica, como acidose láctica em choque séptico, por exemplo. Fitas reagentes para pesquisa de cetonúria também são disponíveis para uso domiciliar. Contudo, o método para pesquisa de cetonúria (com nitroprussiato em fita reagente) não detecta presença de $\mathrm{BHB}$. Como conseqüência disso, durante o tratamento do paciente, a cetonúria pode se acentuar apesar de melhora clínica. Isto se deve a uma diminuição da concentração sérica de $\mathrm{BHB}$ e aumento, durante o período de recuperação da $\mathrm{CAD}$, de sua conversão a acetoacetato, $\mathrm{o}$ qual é detectado pelo método de pesquisa de cetonúria. Logo, esse método é útil para diagnóstico da $\mathrm{CAD}$, mas não para o acompanhamento do seu tratamento.

\section{Uréia e creatinina}

Podem ser elevadas, por desidratação e azotemia prérenal. Além disso, o acetoacetato interfere na medida colorimétrica da creatinina, o que pode levar a creatinina sérica falsamente elevada $(45,46)$. O aumento da proteólise também pode contribuir para aumentar a uréia plasmática.

\section{Hemograma completo}

O hematócrito pode se elevar por hemoconcentração e desidratação. Geralmente há leucocitose, possivelmente acompanhada de desvio para esquerda, mas contagem leucocitária acima de $25.000-30.000 / \mathrm{mm}^{3}$ é sugestiva de infecção.

\section{Amilase e CPK}

Podem estar elevadas, mas o fato parece estar relacionado à interferência de $\mathrm{CC}$ na dosagem e, no caso da amilase, pela liberação extra-pancreática (ex.: pelas parótidas). A elevação da amilase pode, ainda, ser devida à redução de seu clearance, como no caso de insuficiência renal.

\section{EAS}

Além de demonstrar cetonúria, pode revelar alterações sugestivas de infecção urinária (possível fator desencadeante da CAD), cilindros hialinos ou granulosos, aumento de densidade urinária e albuminúria.

\section{Sódio $\left(\mathrm{Na}^{+}\right)$}

$\mathrm{O} \mathrm{Na}{ }^{+}$corporal total está diminuído por perda urinária desse íon. A concentração plasmática, por outro lado, pode ser elevada ou diminuída. Concentração elevada significa perda de água superior à perda de sódio e concentração diminuída pode ser dilucional ou por hiperglicemia e seu efeito osmótico. A concentração plasmática deve ser corrigida de acordo com a glicemia, para interpretação correta: para cada 100 $\mathrm{mg} / \mathrm{dl}$ de glicose acima de $100 \mathrm{mg} / \mathrm{dl}$ adiciona-se 1,6 $\mathrm{mEq}$ à natremia. Alterações de concentração de sódio apresentam maior importância em crianças, já que nestes indivíduos há maior tendência a desenvolvimento de edema cerebral por hiponatremia.

\section{Potássio (K+)}

$\mathrm{O} \mathrm{K}^{+}$corporal total é sempre diminuído (em cerca de 5 $\mathrm{mEq} / \mathrm{kg}$ ), mas a concentração sérica do íon pode ser normal, alta ou baixa. A hipocalemia é considerada o distúrbio eletrolítico com maior risco de vida durante o tratamento da CAD. A diminuição do potássio total se deve à perda urinária desse íon, embora a presença de vômitos também desempenhe seu papel na gênese do processo (10). O hiperaldosteronismo secundário relacionado à depleção de sódio e a presença de cetoácidos de carga negativa no líquido tubular aumentam ainda mais as perdas urinárias de potássio. Se ocorrer hipercalemia, esta se deve a deslocamento de potássio do meio intracelular para o extracelular devido à saída de água do interior das células por hiperglicemia (efeito osmótico), acidose metabólica e proteólise. Além disso, a insulinopenia diminui a entrada de íon potássio nas células, provocando permanência destes no meio extracelular.

\section{Gasometria arterial}

Demonstra acidose metabólica, com diminuição do pH sanguíneo e do bicarbonato sérico. Se o pH é muito baixo e a cetonúria apenas moderada, a causa pode ser acidose láctica, acidose hiperclorêmica ou proporção de BHB muito elevada em relação ao acetoacetato. A dosagem de lactato sérico pode ser útil nestes casos, para melhor esclarecimento. Esse exame também pode ser utilizado como um meio indireto de diagnóstico de CAD, já que lactato sérico baixo associado a acidose metabólica e hiperglicemia é altamente sugestivo desta condição (47). 


\section{Osmolaridade plasmática}

Está sempre elevada e é diretamente proporcional à queda do nível de consciência. Estudos indicam que osmolaridade plasmática superior a $330 \mathrm{mOsm} / \mathrm{kg}$ é associada a torpor e coma. Sua medida pode ser feita com osmômetro ou através da seguinte fórmula:

$2\left(\mathrm{Na}^{+}+\mathrm{K}^{+}\right)+$glicemia $(\mathrm{em} \mathrm{mg} / \mathrm{dl}) / \mathrm{l} 8+$ uréia $(\mathrm{em}$ $\mathrm{mg} / \mathrm{dl}) / 6$

(Normal: 285-295 mOsm $/ \mathrm{kg}$ )

\section{Cálculo do anion GAP}

$\mathrm{O}$ anion gap (soma dos cátions diminuída da soma dos ânions) é aumentado na CAD e pode ser calculado através da seguinte fórmula:

$\mathrm{Na}^{+}-\left(\mathrm{Cl}^{-}+\mathrm{HCO}_{3}^{-}\right)$

(Normal: 7-9) $(48,49)$

A diminuição do anion gap ao longo do tratamento, com persistência da acidose, pode indicar acidose hiperclorêmica (por sobrecarga de cloreto administrado com a solução salina a $0,9 \%$ ).

\section{ECG}

Deve ser solicitado de rotina, para pesquisa de IAM, sobretudo nos pacientes com muitos anos de evolução de DM, em quem o IAM pode ser atípico. IAM pode ser fator desencadeante ou complicação da CAD. O ECG também é útil para acompanhar alterações de concentração sérica de potássio e sua reposição (10).

\section{Rastreamento de infecção}

Também deve ser feito de rotina, na tentativa de identificar fatores precipitantes, com coleta de culturas para bactérias, radiografia de tórax (10) e, se necessário, radiografia ou, preferencialmente, tomografia computadorizada (TC) de seios da face. Lesões de pele e úlceras de decúbito também devem ser pesquisadas (50).

\section{TRATAMENTO}

A cetoacidose leve a moderada pode ser tratada fora de unidade intensiva. Casos mais graves devem ser conduzidos em CTI. A CAD pode ser classificada quanto à gravidade, utilizando-se critérios como bicarbonato sérico, pressão arterial, anion gap, excesso de base e osmolaridade sérica (51). Na tabela 1 estão alguns critérios que auxiliam na diferenciação da gravidade da CAD (27). Em todos casos, é necessária hidratação, insulinoterapia e correção de possíveis anormalidades hidro-eletrolíticas.

\section{Cetoacidose leve (figura 3A)}

Muitas vezes pode ser tratada ambulatorialmente. A hidratação pode ser feita por via oral, desde que esta seja possível. Se houver vômitos freqüentes ou sinais clínicos de desidratação, a administração de fluidos deve ser feita por via parenteral (endovenosa). Nesse caso, a internação pode ser necessária.

$\mathrm{Na} C A D$ leve, pode-se administrar insulina regular ou análogos de insulina de ação ultra-rápida (lispro ou aspart) por via subcutânea a cada uma hora. Tal conduta nesse tipo de paciente é igualmente efetiva à administração venosa de insulina na correção da glicemia e na redução da cetonemia, mas pode apresentar, no caso do uso dos análogos de insulina, um custo superior à terapia convencional (52-55). No caso do uso da insulina regular, deve-se administrar uma dose inicial em bolus de 0,4 a $0,6 \mathrm{UI} / \mathrm{kg}$, sendo metade por via venosa e a outra metade por via subcutânea ou intramuscular (56). A seguir, a dose de manutenção recomendada é a de 0,1 $\mathrm{UI} / \mathrm{kg} / \mathrm{h}$ por via subcutânea ou intramuscular, até que haja correção da glicemia e da desidratação (27). No caso do uso dos análogos de insulina de ação ultra-rápida, a dose recomendada é de $0,3 \mathrm{UI} / \mathrm{kg}$ em bolus por via subcutânea, seguida por uma dose de manutenção de 0,1 $\mathrm{UI} / \mathrm{kg} /$ hora pela mesma via. Essa dose deverá ser mantida até que a desidratação e a hiperglicemia sejam corrigidas $(53,54)$.

\section{Cetoacidose moderada a grave (figura 3B)}

\section{Hidratação}

É o passo mais importante do tratamento. Diminui a retenção de glicose e CC no plasma, além de diminuir o estímulo adrenérgico, a resistência periférica à insulina e o estímulo a gliconeogênese hepática. Alguns

Tabela 1. Critérios diagnóstico e de gravidade na CAD.

\begin{tabular}{lccc}
\hline & CAD leve & CAD moderada & CAD grave \\
\hline pH arterial & $7,25-7,30$ & $7,0-7,24$ & $<7,0$ \\
Bicarbonato sérico $(\mathrm{mEq} / \mathrm{l})$ & $15-18$ & $10 \mathrm{a} 14,9$ & $<10$ \\
Anion gap & $>10$ & $>12$ & $>12$ \\
Nivel sensorial & Alerta & Alerta/sonolento & Estupor/coma \\
\hline
\end{tabular}

Adaptado de Kitabchi e cols., 2004 (27). 
A

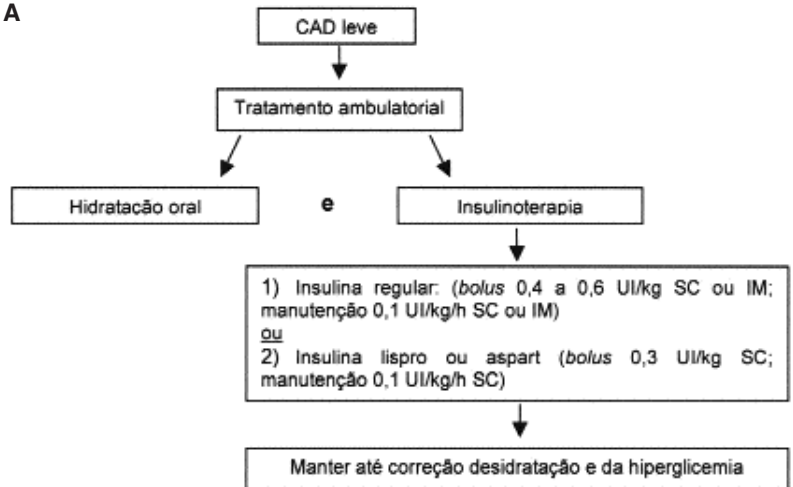

Figura 3. Tratamento da cetoacidose em adultos. A) Tratamento CAD leve.

estudos demonstraram que o retardo do início da insulinoterapia, na vigência de hidratação adequada, não altera de forma significativa a mortalidade na CAD, o que reforça a importância da hidratação. Alguns trabalhos sugerem, ainda, que o início de insulinoterapia após início da hidratação torna as células mais responsivas à insulina administrada (57).

A perda de líquido na CAD é de cerca de 5 a $10 \%$ do peso corporal (10) e a gravidade da desidratação pode ser estimada através da concentração corrigida de sódio e da osmolaridade sérica. As natremias corrigidas superiores a $140 \mathrm{mEq} / \mathrm{l}$ e as osmolaridades superiores a $340 \mathrm{mOsm} / \mathrm{kg}$ associam-se a maior déficit de líquido. A elevação da osmolaridade é relacionada à diminuição do nível de consciência, observando-se tipicamente torpor e coma com osmolaridades de, no mínimo, $330 \mathrm{mOsm} / \mathrm{kg}$ (33).

A reposição de líquidos deve ser generosa, podendo ser feita de várias maneiras e, em pacientes idosos e/ou cardiopatas, deve ser acompanhada de monitorização através de cateterização de artéria pulmonar. Uma conduta proposta é a infusão de solução salina a $0,9 \%$ num volume de aproximadamente 1.000 a $1.500 \mathrm{ml} / \mathrm{h}$ nas primeiras 2 horas $(15 \mathrm{a} 20 \mathrm{ml} / \mathrm{kg}$ ) e de cerca de $500 \mathrm{ml} / \mathrm{h}(4 \mathrm{a} 14 \mathrm{ml} / \mathrm{kg}$, em média 7,5 $\mathrm{ml} / \mathrm{kg}$ ) nas 2 horas subseqüentes, com alterações de acordo com o quadro clínico do paciente (10). Se a osmolaridade for maior do que $320 \mathrm{mOsm} / \mathrm{kg}$ ou o sódio sérico for maior do que $155 \mathrm{mEq} / \mathrm{l}$, a solução salina a $0,45 \%$ (hipotônica) deve substituir a solução a $0,9 \%(58)$.

Quando a glicemia atingir níveis menores do que $250 \mathrm{mg} / \mathrm{dl}$, adiciona-se solução intravenosa de glicose a 5\% (SG5\%), numa velocidade de cerca de 150 a $250 \mathrm{ml} / \mathrm{h}$. O SG5\% deve ser mantido até correção da acidose e normalização do anion gap (10). Alguns autores sugerem uso de frutose a $5 \%$ ao invés de SG5\%,

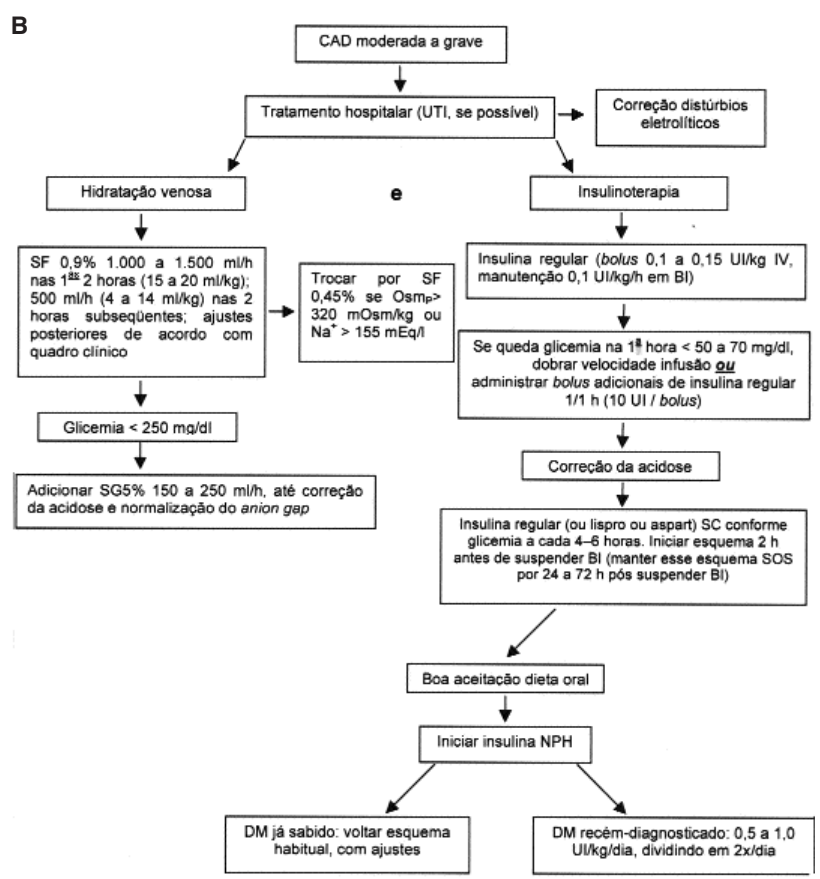

Figura 3. Tratamento da cetoacidose em adultos. B) Tratamento CAD moderada a grave.

$\mathrm{CAD}=$ cetoacidose diabética, $\mathrm{SC}=$ subcutâneo, IV = intravenoso, IM = intramuscular, UTI = unidade de terapia intensiva, SF = soro fisiológico, SG = soro glicosado, DM = diabetes mellitus.

pois a frutose não necessita de insulina para captação celular, mas essa substância pode estimular a produção de lactato. $\mathrm{O}$ uso de solução hipotônica aumenta o fornecimento de água livre e reduz a chance de hipernatremia. Alguns não recomendam seu uso na CAD, pois poderia provocar hemólise e, simultaneamente com a diminuição da glicemia e da cetonemia, levar a quedas rápidas de osmolaridade, tendo como conseqüência o desenvolvimento de edema cerebral.

\section{Insulinoterapia}

Atualmente, a maioria dos autores preconiza uso de baixas doses de insulina no tratamento da CAD, já que esta conduta se mostrou tão eficaz quanto a administração de altas doses de insulina, indicada no passado $(59,60)$. Recomenda-se uma dose inicial de 0,1 a 0,15 UI de insulina regular EV em bolus, seguida de 0,1 $\mathrm{UI} / \mathrm{kg} / \mathrm{h}$ em bomba de infusão por via endovenosa $(10,27)$. A solução para a bomba infusora pode ser preparada com 25 UI de insulina regular (menos de meio $\mathrm{ml}$ ) em $250 \mathrm{ml}$ de solução salina a $0,9 \%$. Nessa solução, cada $10 \mathrm{ml}$ correspondem a 1 UI de insulina. Deve-se desprezar 30 a $50 \mathrm{ml}$ iniciais, que permanecem ligados ao equipo de soro e não são realmente infundidos $(10,61)$. 
A infusão contínua, se não disponível, pode ser substituída por insulina regular por via intramuscular ou infusão venosa intermitente. Contudo, a via intramuscular de administração tem vários inconvenientes. Inicialmente, enquanto o paciente estiver desidratado, ela apresenta uma absorção mais lenta, o que faz com que a queda da glicemia seja mais demorada nesse período. Com a melhora da hidratação, ocorre um aumento da absorção da insulina administrada por essa via, o que pode acarretar uma queda súbita da glicemia. Sendo assim, é difícil prever a velocidade com que a insulina atinge efetivamente a circulação sistêmica quando for administrada por essa forma alternativa.

Caso não haja queda da glicemia capilar em cerca de 50 a $70 \mathrm{mg} / \mathrm{dl}$ na primeira hora, a dose de insulina deve ser dobrada nas horas subseqüentes até que essa meta de queda glicêmica seja atingida. Outra opção, caso não haja a queda esperada da glicemia, é a administração de bolus adicionais de insulina a cada hora (cerca de 10 UI em cada bolus após a dose de ataque) (27).

Após negativação da cetonúria e normalização do pH sanguíneo, a infusão contínua deve ser substituída por insulina de ação rápida (insulina regular) ou ultrarápida (lispro ou aspart), sendo a primeira mais freqüentemente utilizada, por via subcutânea a cada 4 a 6 horas, conforme esquema pré-estabelecido. Tal esquema deve ser mantido nas primeiras 24 a 72 horas após a suspensão da infusão venosa contínua. A primeira dose da insulina de ação rápida subcutânea deve ser administrada 2 horas antes da suspensão da infusão venosa contínua, uma vez que a insulina subcutânea demora mais do que a venosa para iniciar seu efeito, mesmo com o uso das insulinas ultra-rápidas. Deve-se lembrar que, em determinadas situações, a dose de insulina deve ser reduzida, pelo elevado risco de hipoglicemia, como é o caso da insuficiência renal e do primeiro trimestre de gestação. Em contraposição, outras situações tornam necessárias altas doses de insulina rápida ou ultra-rápida, como é o caso da presença de infecções, do uso de corticóides e de pacientes na adolescência (10).

Insulina de ação intermediária (NPH, lenta) ou análogo de insulina de longa duração (detemir, glargina) deve ser introduzida após início de aceitação alimentar.

Nos pacientes que já faziam uso de insulina, deve-se reintroduzir a mesma dose previamente utilizada, fazendo os ajustes apropriados. No caso de paciente recém diagnosticado, deve-se administrar uma dose inicial de insulina de 0,5 a 1,0 UI/ $\mathrm{kg} / \mathrm{dia}$, fracionando em pelo menos duas tomadas diárias, em terapia basal-bolus. Administração de insulina de ação rápida antes das refeições, de acordo com os níveis glicêmicos, deve ser mantida. Alguns pacientes com DM2 podem receber alta em uso de hipoglicemiantes orais, após compensação clínica (62).

Em casos selecionados (pacientes com resistência insulínica significativa por altos títulos de anticorpos anti-insulina ou outros fatores), são necessárias altas doses de insulina para o tratamento da CAD, com bolus inicial de 50 UI seguido de 10 a 20 UI em infusão contínua por hora. O uso de altas doses de insulina pode, ainda, ser iniciado em pacientes sem resposta ao tratamento com baixas doses de insulina nas 3 ou 4 primeiras horas (ausência de aumento do $\mathrm{pH}$ ou redução do anion gap), pela possibilidade de resistência a insulina nesses casos $(63,64)$.

O tempo necessário para que o paciente atinja os valores laboratoriais pré-determinados de glicemia, bicarbonato e $\mathrm{pH}$ varia de acordo com os níveis inicialmente encontrados e com a presença de outras patologias desencadeantes. A glicemia deve ser reduzida em 75 a $100 \mathrm{mg} / \mathrm{dl} / \mathrm{h}$. De uma maneira geral, como uma dose fisiológica de insulina reduz a glicemia a uma velocidade de $80 \mathrm{mg} / \mathrm{dl} / \mathrm{h}$, admite-se que o tempo para que o paciente atinja glicemia de 200 $\mathrm{mg} / \mathrm{dl}$, após início do tratamento adequado, pode ser calculado pela fórmula a seguir:

Glicemia inicial $(\mathrm{mg} / \mathrm{dl})-200 / 80$

Calcula-se que o tempo necessário para controle dos níveis de $\mathrm{pH}$ e bicarbonato seja o dobro do que a glicemia leva para atingir $200 \mathrm{mg} / \mathrm{dl}$. Pacientes torporosos e com osmolaridade elevada necessitam do mesmo número de horas para recuperar a consciência e para normalizar $\mathrm{pH}$ e bicarbonato. Nesses pacientes, alguns trabalhos recomendam que a glicose não seja reduzida para menos de $250 \mathrm{mg} / \mathrm{dl}$, mas mantida neste nível, até que o paciente esteja lúcido e orientado $(2,27,33,62,65,66)$.

Deve-se ter atenção para que não ocorra queda da osmolaridade plasmática acima de $3 \mathrm{mOsm} / \mathrm{kg} / \mathrm{h}$, devido ao risco do surgimento de edema cerebral $(31,32,56,67)$, conforme será detalhado adiante.

\section{Correção dos distúrbios eletrolíticos}

Potássio: A administração de potássio na CAD deve ser sempre considerada, pois o potássio corporal total é invariavelmente baixo. O déficit total é de 300-1.000 mEq/l. Pacientes com concentração sérica de potássio normal ou baixa na admissão apresentam as maiores deficiências de potássio e podem apresentar hipocalemia severa após início da insulinoterapia, que irá deslocar o potássio do meio extracelular para o intracelular. A reposição de potássio deve ser iniciada 
Tabela 2. Dose de reposição de potássio no tratamento da CAD.

\begin{tabular}{lcc}
\hline Potássio plasmático & $\begin{array}{c}\text { Dose a ser administrada } \\
(\text { em } \mathrm{mEq} / \mathrm{Kg} / \mathrm{h})\end{array}$ & $\begin{array}{c}\text { Dose a ser administrada } \\
(\mathrm{em} / \mathrm{Kg} / \mathrm{h})\end{array}$ \\
$<3 \mathrm{mEq} / \mathrm{l}$ & $\geq 0,6 \mathrm{mEq} / \mathrm{Kg} / \mathrm{h}$ & $\geq 0,044 \mathrm{~g} / \mathrm{Kg} / \mathrm{h}$ \\
$3-4 \mathrm{mEq} / \mathrm{l}$ & $0,6 \mathrm{mEq} / \mathrm{Kg} / \mathrm{h}$ & $0,044 \mathrm{~g} / \mathrm{Kg} / \mathrm{h}$ \\
$4-5 \mathrm{mEq} / \mathrm{l}$ & $0,2-0,4 \mathrm{mEq} / \mathrm{Kg} / \mathrm{h}$ & $0,01-0,03 \mathrm{~g} / \mathrm{Kg} / \mathrm{h}$ \\
$5-6 \mathrm{mEq} / \mathrm{l}$ & $0,1-0,2 \mathrm{mEq} / \mathrm{Kg} / \mathrm{h}$ & $0,007-0,01 \mathrm{~g} / \mathrm{Kg} / \mathrm{h}$ \\
$>6 \mathrm{mEq} / \mathrm{l}$ & Suspender infusão de $\mathrm{K}+$ até que & Suspender infusão de $\mathrm{K}+$ até que \\
& os níveis caiam para $<6 \mathrm{mEq} / \mathrm{l}$ & os níveis caiam para $<6 \mathrm{mEq} / \mathrm{l}$ \\
\hline
\end{tabular}

Adaptado de Medical Management of Type I Diabetes, 2004 (10).

assim que o paciente apresentar a primeira diurese. A quantidade de potássio a ser administrada é estimada em função dos níveis plasmáticos desse íon, conforme esquematizado na tabela 2 .

$1 \mathrm{~g}$ potássio $=13,5 \mathrm{mEq}$

Por exemplo, em um indivíduo com cerca de 66 $\mathrm{kg}$, a conduta a ser tomada seria: se $\mathrm{K}^{+}<3 \mathrm{mEq} / \mathrm{l}$ : administrar cerca de $40 \mathrm{mEq} / \mathrm{h}(3 \mathrm{~g} / \mathrm{h})$, ou seja, 30 $\mathrm{ml}$ de $\mathrm{KCl} 10 \%$.

A velocidade de infusão de potássio não deve exceder $40 \mathrm{mEq} / \mathrm{h}$. O objetivo do tratamento é manter o potássio sérico entre 4 e $5 \mathrm{mEq} / \mathrm{l}$. Recomenda-se monitorização eletrocardiográfica durante a reposição desse eletrólito. Alguns autores acreditam que a insulinoterapia deva ser iniciada somente após a instituição da reposição de potássio, levando em consideração que a insulina é um dos mecanismos mais poderosos para deslocamento do potássio para o meio intracelular (33). Em pacientes que apresentem exame admissional com hipocalemia grave $(<3,3 \mathrm{mEq} / \mathrm{l})$, pode-se iniciar a reposição de potássio juntamente com a hidratação venosa, postergando o início da insulinização até que os níveis desse eletrólito estejam $>3,3 \mathrm{mEq} / 1$. Tal conduta se baseia no fato de que graus tão severos de hipocalemia oferecem risco de complicações, como arritmias cardíacas, parada cárdio-respiratória ou ainda de fraqueza da musculatura respiratória, com potencial evolução para insuficiência respiratória aguda (27).

Bicarbonato: Deve ser reposto somente se $\mathrm{pH}$ for $<7,0$, pois a administração de bicarbonato pode piorar a hipocalemia $(68,69)$, produzir acidose paradoxal do líquor no sistema nervoso central $(68,70,71)$, piorar a acidose intracelular (por maior produção de dióxido de carbono) $(15,72,73)$, causar hipocalcemia $(68,70,71)$ e hipóxia $(68,74)$ e alentecer a queda da cetonemia (75). Existem evidências de que a terapia com bicarbonato pode aumentar a síntese de corpos cetônicos, contrapondo-se, assim, ao efeito da insulina. A solução utilizada para correção da acidose é bicarbonato a $8,4 \%$, e a dose é calculada de acordo com o cálculo do déficit de bases (DB):
$\mathrm{DB}(\mathrm{mEq})=0,5$ peso corporal $(\mathrm{kg}) \mathrm{X}\left(24-\mathrm{HCO}_{3}{ }^{-}\right)$ $1 \mathrm{ml} \mathrm{NaHCO} 38,4 \%=1 \mathrm{mEq}$ de $\mathrm{HCO}_{3}{ }^{-}$

Deve-se administrar $50 \%$ do $\mathrm{DB}$ calculado, sendo metade dessa parte ( $1 / 4$ do total) infundida em 2 horas e a outra metade ( $1 / 4$ do total) de forma contínua, em 4 a 6 horas, por via endovenosa.

Fosfato $\left(\mathrm{PO}_{4}\right)$ : Apesar de haver um déficit de fosfato de cerca de $1 \mathrm{mmol} / \mathrm{kg}$ durante a CAD, os exames mostram níveis séricos normais ou ainda elevados desse íon na admissão dos pacientes acometidos (27). A administração de fosfato é controversa, mesmo que sua concentração sérica seja baixa, pois alguns autores acreditam que os valores retornem à níveis adequados com normalização do $\mathrm{pH}$ e aumento da utilização periférica de glicose (a acidose aumenta excreção renal de fosfato e a diminuição da utilização periférica de glicose diminui a captação celular de fosfato). $\mathrm{O}$ fosfato sérico em baixas concentrações, entretanto, desvia a curva de oxi-hemoglobina para a esquerda, provocando retenção de $\mathrm{O}_{2}$ pelas hemácias. Além disso, foi verificada melhora da função mental e menor mortalidade com reposição de fosfato (76). Possíveis indicações para uso de fosfato seriam disfunção ventricular esquerda, ausência de melhora do estado mental apesar de melhora da acidose, hiperosmolaridade, anemia, depressão respiratória ou fosfato sérico abaixo de $1 \mathrm{mg} / \mathrm{dl}$. No caso da necessidade de reposição, a dose proposta é de até 1,5 $\mathrm{mEq} / \mathrm{Kg}$ por via endovenosa em 24 horas (10). A reposição de fosfato pode ser feita na forma de $\mathrm{KPO}_{4}$ durante a reposição de potássio $(2 / 3$ da reposição de potássio poderia ser realizada com $\mathrm{KCl}$ e $1 / 3$ com $\mathrm{KPO}_{4}$ ) (27). Entretanto, devemos atentar para os riscos decorrentes da sua utilização, como o surgimento de hipocalcemia grave sem os sinais clínicos evidentes de tetania (77).

Magnésio: Apesar de também ser depletado, não se sabe a relevância clínica de sua deficiência. Se magnésio for $<1,8 \mathrm{mEq} / 1$ ou houver tetania, sulfato de magnésio deve ser administrado. A dose recomendada nessas situações é de $5 \mathrm{~g}$ de sulfato de magnésio em $500 \mathrm{ml}$ de solução salina a $0,45 \%$ em 5 horas. 
Cálcio: perda de cálcio pode ocorrer, mas a reposição de cálcio somente está indicada na hipocalcemia sintomática.

Sódio: A reposição de sódio é feita com a administração de solução salina a $0,9 \%$.

\section{AVALIAÇÃO INICIAL E ACOMPANHAMENTO DO PACIENTE}

A avaliação inicial deve ser feita com bioquímica do sangue, glicemia capilar, pesquisa de cetonúria/ dosagem de cetonemia, hemograma completo, gasometria arterial e EAS. Em seguida, inicia-se infusão de solução salina a $0,9 \%$, enquanto a realização de outros exames é iniciada (culturas, ECG). A rotina proposta para seguimento dos pacientes durante o tratamento da CAD está esquematizada na tabela 3.

Não há necessidade de coleta de sangue arterial para dosagem de $\mathrm{pH}$ e bicarbonato para monitorização do tratamento, pois a coleta de sangue venoso é mais simples e menos dolorosa. $\mathrm{O} \mathrm{pH}$ do sangue venoso deve ser corrigido para interpretação correta: usualmente é 0,03 mais baixo do que o $\mathrm{pH}$ arterial (47).

Outras medidas gerais devem ser tomadas a fim de evitar complicações clínicas no paciente. As vias aéreas devem ser mantidas pérveas, tendo-se cuidado para a possibilidade de aspiração de conteúdo gástrico em pacientes com redução do nível de consciência. Nesses casos, em função da gastroparesia apresentada durante 0 quadro de $\mathrm{CAD}$, recomenda-se a inserção de um tubo nasogástrico para drenagem do conteúdo gástrico, para prevenção de pneumonia por aspiração. Baixas doses de heparina subcutânea (5.000 UI SC a cada 12 horas) para profilaxia de complicações trombóticas são geralmente recomendadas, principalmente nos mais idosos. Contudo, até o momento, não existem estudos que comprovem o benefício do uso profilático dessa medicação nessa população específica (10).

O paciente deve ser orientado que, mesmo após a resolução do quadro agudo e a sua alta hospitalar, a resistência insulínica pode persistir por algumas semanas, de modo que a dose de insulina necessária nesse período pode ser superior à utilizada pelo paciente previamente ao surgimento dessa descompensação aguda (10).

\section{ABORDAGEM DO PACIENTE COM CAD EM USO DE BOMBA DE INSULINA}

Os indivíduos em uso de BISCI com hiperglicemia importante $(>250 \mathrm{mg} / \mathrm{dL}$ ) devem ser orientados a pesquisar cetonúria e/ou de cetonemia (em teste capilar). Caso a cetonúria esteja +2 ou mais e/ou a cetonemia estiver $>1 \mathrm{mmol} / \mathrm{L}$, o paciente deve administrar um bolus subcutâneo de insulina de ação rápida (ou ultra-rápida) com seringa (ou caneta), calculada a partir do cálculo do fator de sensibilidade (FS, que é igual a 1800/dose total diária de insulina). Outra opção é a administração de $20 \%$ da dose total diária de insulina em uso. A escolha entre essas duas formas de cálculo deve ser a favor daquela que proporcionar a maior quantidade de insulina a ser ofertada.

Após o bolus, deve-se checar a glicemia capilar em 2 horas. Caso haja queda da glicemia, deve-se manter essa monitorização a cada 2 horas até normalização da glicemia capilar. Caso não ocorra queda da glicemia, o médico assistente deve ser prontamente contatado.

\section{COMPLICAÇÕES}

As complicações mais freqüentes da CAD são a hipoglicemia, a hipocalemia e a hiperglicemia (27).

\section{Hipoglicemia}

Pode ser o resultado da administração excessiva de insulina durante o tratamento inicial ou da manutenção da infusão de insulina sem a reposição concomitante de SG5\% após a obtenção de glicemias క $250 \mathrm{mg} / \mathrm{dl}(27)$.

Tabela 3. Exames no acompanhamento da CAD.

\begin{tabular}{ll}
\hline EXAME & FREQÜÊNCIA \\
\hline Glicemia capilar & A cada 1 hora \\
Glicemia plasmática & A cada 2 -4 horas \\
Cetonúria & A cada 4 horas \\
$\mathrm{K}^{+}$ & Horária nas primeiras $4 \mathrm{~h}$ e de a cada 2-4 horas em seguida \\
$\mathrm{Na}^{+}, \mathrm{Cl}^{-}, \mathrm{HCO}_{3}^{-}$(anion gap) e pH sangüíneo & A cada $2 \mathrm{~h}$ até normalização do potássio e do bicarbonato, \\
$\mathrm{Mg}^{++}$, Fosfato & depois a cada 4 a 6 h até recuperação plena \\
\hline
\end{tabular}

Adaptado de Medical Management of Type I Diabetes, 2004 (10). 


\section{Hipocalemia}

Decorrente da terapia com insulina e do tratamento da acidose com bicarbonato sem os cuidados recomendados anteriormente (27).

\section{Hiperglicemia a cetoacidose reentrante}

Trata-se geralmente de uma complicação secundária ao manejo inadequado da fase de transição da administração de insulina por via endovenosa contínua para a via subcutânea em múltiplas doses diárias. Nesse caso, o paciente em recuperação pode voltar a apresentar acidose e hiperglicemia, voltando a necessitar de insulinização venosa contínua $(10,27)$.

\section{Edema cerebral}

É uma complicação rara, sendo mais freqüente em crianças do que em adultos. Apesar de incomum, muitas vezes é fatal (10). Pode ocorrer por acidose paradoxal do líquor durante o tratamento com bicarbonato de sódio (já que este não penetra no líquido céfalo-raquidiano), por diminuição da oxigenação do sistema nervoso central (SNC), por efeito direto da insulina no transporte de água através de membrana e por produção de osmóis idiogênicos (possivelmente mioinositol, taurina e betaína). Os osmóis idiogênicos são substâncias produzidas pelos neurônios visando impedir sua retração, ou seja, para manter seu volume, durante a hiperosmolaridade extracelular provocada pela glicemia. A metabolização dos osmóis idiogênicos é mais lenta do que a queda da osmolaridade, o que pode gerar influxo de líquidos para o interior dos neurônios, causando edema cerebral. Além disso, a própria insulinoterapia promove entrada de partículas osmoticamente ativas para o espaço intracelular. A reposição muito rápida de sódio também pode contribuir para formação de edema cerebral (78). A correção gradativa do déficit de sódio e de água e os cuidados para evitar a queda rápida da glicemia são importantes medidas preventivas desta complicação $(10,78)$. As principais manifestações clínicas podem surgir rapidamente, com deterioração do nível de consciência, alterações do comportamento, letargia e cefaléia, evoluindo com convulsões, alterações pupilares, bradicardia, incontinência urinária e parada respiratória, em função de herniação do tronco encefálico $(27,79)$. Estudos recentes têm sugerido que haja um papel preponderante de um mecanismo vasogênico do que o mecanismo citotóxico acima mencionado. Tais hipóteses baseiam-se em modelos animais, nos quais evidências sugerem que ocorra ativação de canais iônicos na barreira hemato-encefálica, o que contribuiria com o edema cerebral. A ativação de tais canais poderia ser secundária à isquemia cerebral, a um efeito direto das cetonas na barreira hemato-encefálica e/ou à ação de citocinas inflamatórias presentes durante a CAD (80-82).

\section{Acidose hiperclorêmica}

Não tem efeitos clínicos adversos e é gradativamente corrigida em 24 a 48 horas, por aumento de excreção renal de ácidos (50). É causada pela infusão de líquidos intravenosos contendo cloro em concentrações superiores às plasmáticas, pela expansão de volume com líquidos sem bicarbonato e pelos desvios intracelulares de bicarbonato durante correção da CAD. A acidose hiperclorêmica é mais freqüente nas primeiras 2 a 4 horas de tratamento.

\section{Outras complicações}

Infecções intercorrentes (como Mucormicose, papilite necrotizante e otite externa maligna), necrose tubular aguda, choque (principalmente hipovolêmico), Síndrome de angústia respiratória do adulto (SARA), coagulação intravascular disseminada, IAM, AVC (83).

\section{PREVENÇÃo}

Tão importante quanto um tratamento apropriado é a prevenção da $\mathrm{CAD}$, uma vez que, na maioria das vezes, trata-se de uma complicação que pode ser prevenida pela orientação adequada do paciente. Todos os indivíduos com DM devem realizar a monitorização domiciliar da glicemia capilar regularmente e ser orientados a realizar testes para pesquisa de cetonas na urina ou sangue caso haja hiperglicemia persistente $(\geq 300 \mathrm{mg} / \mathrm{dl}$ ou 250 $\mathrm{mg} / \mathrm{dl}$, em casos selecionados, especialmente em vigência de doenças intercorrentes, como as infecções). Nesses casos, o paciente deve corrigir a hiperglicemia com a administração suplementar de insulina, aumentar a ingesta hídrica e monitorizar cuidadosamente a glicemia capilar no período subseqüente. Caso haja manutenção da cetonemia/cetonúria e da hiperglicemia, o paciente deve contatar seu médico assistente (10).

Os pacientes devem ser orientados para que, caso estejam vomitando e não consigam se alimentar, procurem atendimento médico de urgência e não devem omitir a dose de insulina por medo de hipoglicemia, o que pode levar a um quadro de CAD em algumas horas (10).

\section{DIAGNÓSTICO DIFERENCIAL}

Algumas situações devem ser diferenciadas dos achados clínicos e laboratoriais da CAD. A cetose de jejum e a cetoacidose alcoólica são diferenciadas pela história 
clínica de jejum e de abuso alcoólico, respectivamente, e pela glicemia plasmática, que pode estar pouco elevada (raramente $>250 \mathrm{mg} / \mathrm{dl}$ ), ou até em níveis muito baixos. A cetoacidose alcoólica pode apresentar-se com acidose importante, enquanto que a cetose de jejum geralmente não cursa com níveis de bicarbonato abaixo de $18 \mathrm{mEq} / \mathrm{l}(27)$.

As outras causas de acidose metabólica com anion gap elevado também devem ser consideradas no diagnóstico diferencial da CAD: acidose láctica, ingestão de drogas (salicilato, metanol, etilenoglicol e paraldeído) e insuficiência renal crônica. Em casos de dúvida, pode-se realizar a dosagem de lactato, salicilato e metanol séricos. A intoxicação por etilenoglicol pode ser suspeitada pela presença de oxalato de cálcio e cristais de hipurato no exame de urina (27).

\section{MORTALIDADE E PERSPECTIVAS FUTURAS}

A mortalidade na CAD geralmente não é resultado da hipertonicidade ou acidose, e sim por fatores concomitantes que precipitaram o distúrbio ou se desenvolveram durante o tratamento (ex.: IAM, sepse, pancreatite) (38). Na maioria dos serviços essa taxa é de 7 a $11 \%$. No Brasil, existem poucos dados sobre a freqüência dessa evolução adversa, mas dados sugerem que seja similar àquela encontrada em países desenvolvidos (84-86). Com a melhora da qualidade de atendimento médico (maior disponibilidade de exames e treinamento adequado de profissionais de saúde para manejo da condição), houve queda significativa da mortalidade ao longo das últimas décadas, mas a percentagem ainda é superior à desejada. Para que essas taxas continuem em declínio, é fundamental que os conhecimentos sobre esta complicação do DM continuem a ser divulgados, especialmente para especialistas em Terapia Intensiva e Medicina de Urgência.

\section{REFERÊNCIAS}

1. Norris JM, Barriga K, Klingensmith G, Hoffman M, Eisenbarth GS, Erlich HA, et al. Timing of initial cereal exposure in infancy and risk of islet autoimmunity. JAMA 2003;290(13):171320.

2. English P, Williams G. Hyperglycaemic crises and lactic acidosis in diabetes mellitus. Postgrad Med J 2004;80(943):253-61.

3. Sadauskaite-Kuehne V, Samuelsson U, Jasinskiene $E$, Padaiga Z, Urbonaite B, Edenvall $H$, et al. Severity at onset of childhood type 1 diabetes in countries with high and low incidence of the condition. Diabetes Res Clin Pract 2002; 55(3):247-54.
4. Punnose J, Agarwal MM, EI KA, Devadas K, Mugamer IT. Childhood and adolescent diabetes mellitus in Arabs residing in the United Arab Emirates. Diabetes Res Clin Pract 2002;55(1):29-33.

5. Rodacki M, Pereira JR, de Oliveira AM, Barone B, Dowell RM, Perricelli $P$, et al. Ethnicity and young age influence the frequency of diabetic ketoacidosis at the onset of type 1 diabetes. Diabetes Res Clin Pract 2007;78(2):259-62.

6. Rewers A, Chase HP, Mackenzie T, Walravens P, Roback M, Rewers $\mathrm{M}$, et al. Predictors of acute complications in children with type 1 diabetes. JAMA 2002;287(19):2511-8.

7. da Silveira VMF, Menezes AMB, Post CLA, Machado EC. Uma amostra de pacientes com Diabetes tipo 1 no Sul do Brasil. Arq Bras Endocrinol Metab 2001;45(5):433-40.

8. Apud Gomes MB, Aguiar GLK, Marques EP, Sarno EN, Chacra AR. Estudo comparativo de variáveis epidemiológicas em casos múltiplos e esporádicos de Diabetes mellitus do tipo 1. Arq Bras Endocrinol Metab 1996;40:13-5.

9. Campos JJB, Almeida HG, lochida LC, Franco LJ. Incidência de Diabetes Mellitus insulino-dependente (Tipo 1) na cidade de Londrina, PR, Brasil. Arq Bras Endocrinol Metab 1998;42(1):36-44.

10. Medical Management of Type I Diabetes. $4^{\text {th }}$ ed. Alexandria: American Diabetes Association, 2004. pp. 127-35.

11. Kreisberg RA. Diabetic ketoacidosis: new concepts and trends in pathogenesis and treatment. Ann Intern Med 1978;88(5):681-95.

12. DeFronzo RA, Matsuda M, Barrett E. Diabetic ketoacidosis: a combined metabolic-nephrologic approach to therapy. Diabetes Rev 1994;2:209-38.

13. Malone ML, Gennis V, Goodwin JS. Characteristics of diabetic ketoacidosis in older versus younger adults. J Am Geriatr Soc 1992;40(11): $1100-4$.

14. Morris LE, Kitabchi AE. Coma in the diabetic. In: Schnatz JD (ed). Diabetes mellitus: Problems in management. Menlo Park: Addison-Wesley, 1982. pp. 243-51.

15. Krentz AJ, Nattrass M. Acute metabolic complications of diabetes: diabetic ketoacidosis, hyperosmolar non-ketotic hyperglicemia and lactic acidosis. In: Pickup JC, Williams G (ed). Textbook of Diabetes. Oxford: Blackwell Science Ltd, 2003. pp. 1-32.

16. Polonsky WH, Anderson BJ, Lohrer PA, Aponte JE, Jacobson AM, Cole CF. Insulin omission in women with IDDM. Diabetes Care 1994;17(10):1178-85.

17. Banerji MA, Chaiken RL, Huey H, Tuomi T, Norin AJ, Mackay $I R$, et al. GAD antibody negative NIDDM in adult black subjects with diabetic ketoacidosis and increased frequency of human leukocyte antigen DR3 and DR4. Flatbush diabetes. Diabetes 1994;43(6):741-5.

18. Umpierrez GE, Casals MM, Gebhart SP, Mixon PS, Clark WS, Phillips LS. Diabetic ketoacidosis in obese African-Americans. Diabetes 1995;44(7):790-5.

19. Guilhem I, Leguerrier AM, Lecordier F, Poirier JY, Maugendre D. Technical risks with subcutaneous insulin infusion. Diabetes Metab 2006:32(3):279-84.

20. Boland EA, Grey M, Oesterle A, Fredrickson L, Tamborlane WV. Continuous subcutaneous insulin infusion. A new way to lower risk of severe hypoglycemia, improve metabolic control, and enhance coping in adolescents with type 1 diabetes. Diabetes Care 1999;22(11):1779-84.

21. Chantelau E, Spraul M, Muhlhauser I, Gause R, Berger M. Long-term safety, efficacy and side-effects of continuous subcutaneous insulin infusion treatment for type 1 (insulindependent) diabetes mellitus: a one centre experience. Diabetologia 1989;32(7):421-6.

22. Bode BW, Steed RD, Davidson PC. Reduction in severe hypoglycemia with long-term continuous subcutaneous insulin infusion in type I diabetes. Diabetes Care 1996;19 (4):324-7.

23. Pickup J, Keen H. Continuous subcutaneous insulin infusion at 25 years: evidence base for the expanding use of insulin pump therapy in type 1 diabetes. Diabetes Care 2002;25 (3):593-8. 
24. Steindel BS, Roe TR, Costin G, Carlson M, Kaufman FR. Continuous subcutaneous insulin infusion (CSII) in children and adolescents with chronic poorly controlled type 1 diabetes mellitus. Diabetes Res Clin Pract 1995;27(3):199-204.

25. Implementation of treatment protocols in the Diabetes Control and Complications Trial. Diabetes Care 1995;18(3):361-76.

26. Egger M, Davey SG, Stettler C, Diem P. Risk of adverse effects of intensified treatment in insulin-dependent diabetes mellitus: a meta-analysis. Diabet Med 1997;14(11):919-28.

27. Kitabchi $A E$, Umpierrez GE, Murphy MB, Barrett EJ, Kreisberg RA, Malone JI, et al. Hyperglycemic crises in diabetes. American Diabetes Association. Position Statement. Diabetes Care 2004; 27(suppl 1):S94-102.

28. Reichard GA Jr, Skutches CL, Hoeldtke RD, Owen OE. Acetone metabolism in humans during diabetic ketoacidosis. Diabetes 1986;35(6):668-74.

29. Owen OE, Trapp VE, Skutches CL, Mozzoli MA, Hoeldtke RD Boden $\mathrm{G}$, et al. Acetone metabolism during diabetic ketoacidosis. Diabetes 1982;31(3):242-8.

30. Beigelman PM. Severe diabetic ketoacidosis (diabetic "coma"). 482 episodes in 257 patients; experience of three years. Diabetes 1971;20(7):490-500.

31. Marshall SM, Wlaker M, Alberti KGMM. Diabetic ketoacidosis and hyperglycemic non-ketotic coma. In: Alberti KGMM, Zimmet P, DeFronzo RA (ed). International Textbook of Diabetes Mellitus. $2^{\text {nd }}$ ed. New York: John Wiley, 1997. pp. 1215-29.

32. Ennis ED, Stahl EJ, Kreisberg RA. Diabetic ketoacidosis. In: Porte DJ, Sherwin RS (ed). Diabetes Mellitus: Theory and Practice. $5^{\text {th }}$ ed. Amsterdam: Elsevier, 1997. pp. 827-44.

33. Kitabchi AE, Wall BM. Diabetic ketoacidosis. Med Clin North Am 1995;79(1):9-37.

34. Oliveira JEP. Coma Diabético - Cetoacidose, com Hiperosmolar não cetótico e acidose láctica. In: Vaisman $\mathrm{M}$, Tendrich M (ed). Diabetes Mellitus. 1a ed. Editora Cultura Médica; 1994.

35. Matz R. Hypothermia in diabetic acidosis. Hormones 1972;3(1):36-41

36. Kamalakannan D, Baskar V, Barton DM, Abdu TA. Diabetic ketoacidosis in pregnancy. Postgrad Med J 2003;79 (934):454-7.

37. Umpierrez GE, Watts NB, Phillips LS. Clinical utility of betahydroxybutyrate determined by reflectance meter in the management of diabetic ketoacidosis. Diabetes Care 1995;18(1):137-8.

38. Delaney MF, Zisman A, Kettyle WM. Diabetic ketoacidosis and hyperglycemic hyperosmolar nonketotic syndrome. Endocrinol Metab Clin North Am 2000;29(4):683-705.

39. Abrahamson M, Beaser R, Carver C, Cooppan R, Grossman S, Laffel $L$, et al. Guideline for the management of hyperglicemic emergencies for adults. Última atualização: 2004.

40. Orsini-Federici M, Akwi JA, Canonico V, Celleno R, Ferolla P, Pippi $R$, et al. Early detection of insulin deprivation in continuous subcutaneous insulin infusion-treated patients with type 1 diabetes. Diabetes Technol Ther 2006;8(1):67-75.

41. Fabietti PG, Canonico V, Orsini FM, Sarti E, Massi BM. Model based study on monitoring ketone bodies to improve safety in intensive insulin therapy. Int $J$ Artif Organs 2006 29(6):596-601.

42. Guerci B, Benichou M, Floriot M, Bohme P, Fougnot S, Franck $P$, et al. Accuracy of an electrochemical sensor for measuring capillary blood ketones by fingerstick samples during metabolic deterioration after continuous subcutaneous insulin infusion interruption in type 1 diabetic patients. Diabetes Care 2003;26(4):1137-41.

43. Wallace TM, Matthews DR. Recent advances in the monitoring and management of diabetic ketoacidosis. QJM 2004;97(12):773-80.

44. Laffel LM, Wentzell K, Loughlin C, Tovar A, Moltz K, Brink S. Sick day management using blood 3-hydroxybutyrate (3$\mathrm{OHB}$ ) compared with urine ketone monitoring reduces hospital visits in young people with T1DM: a randomized clinical trial. Diabet Med 2006;23(3):278-84
45. Assadi FK, John EG, Fornell L, Rosenthal IM. Falsely elevated serum creatinine concentration in ketoacidosis. J Pediatr 1985;107(4):562-4.

46. Gerard SK, Khayam-Bashi H. Characterization of creatinine error in ketotic patients. A prospective comparison of alkaline picrate methods with an enzymatic method. Am J Clin Pathol 1985;84(5):659-64.

47. Rezende G. Emergências no Diabetes Mellitus. In: Mesquita ET (ed). Emergências Clínico-Cardiológicas. 1a ed. Editora Revinter, 2000.

48. Winter SD, Pearson JR, Gabow PA, Schultz AL, Lepoff RB. The fall of the serum anion gap. Arch Intern Med 1990;150(2):311-3.

49. Sadjadi SA. A new range for the anion gap. Ann Intern Med 1995;123(10):807.

50. Reasner CA, Isley WL. Endocrine emergencies. Recognizing clues to classic problems. Postgrad Med 1997;101(3):231-8, 241.

51. Marinac JS, Mesa L. Using a severity of illness scoring system to assess intensive care unit admissions for diabetic ketoacidosis. Crit Care Med 2000;28(7):2238-41.

52. Fisher JN, Shahshahani MN, Kitabchi AE. Diabetic ketoacidosis: low-dose insulin therapy by various routes. N Engl J Med 1977;297(5):238-41

53. Umpierrez GE, Latif K, Stoever J, Cuervo R, Park L, Freire AX, et al. Efficacy of subcutaneous insulin lispro versus continuous intravenous regular insulin for the treatment of patients with diabetic ketoacidosis. Am J Med 2004:117(5):291-6.

54. Umpierrez GE, Cuervo R, Karabell A, Latif K, Freire AX, Kitabchi AE. Treatment of diabetic ketoacidosis with subcutaneous insulin aspart. Diabetes Care 2004:27(8):1873-8.

55. Della Manna T, Steinmetz L, Campos PR, Farhat SC, Schvartsman $\mathrm{C}$, Kuperman $\mathrm{H}$, et al. Subcutaneous use of a fast-acting insulin analog: an alternative treatment for pediatric patients with diabetic ketoacidosis. Diabetes Care 2005;28(8):1856-61.

56. Kitabchi AE, Sacks HS, Young RT, Morris L. Diabetic ketoacidosis: reappraisal of therapeutic approach. Ann Rev Med 1979;30:339-57

57. Laffel L. Sick-day management in type 1 diabetes. Endocrinol Metab Clin North Am 2000;29(4):707-23.

58. Lebovitz HE. Diabetic ketoacidosis. Lancet 1995;345 (8952):767-72.

59. Kitabchi AE, Ayyagari V, Guerra SM. The efficacy of low-dose versus conventional therapy of insulin for treatment of diabetic ketoacidosis. Ann Intern Med 1976;84(6):633-8.

60. Morris LR, Kitabchi AE. Efficacy of low-dose insulin therapy for severely obtunded patients in diabetic ketoacidosis. Diabetes Care 1980;3(1):53-6.

61. Magee MF, Bhatt BA. Management of decompensated diabetes. Diabetic ketoacidosis and hyperglycemic hyperosmolar syndrome. Crit Care Clin 2001;17(1):75-106.

62. Kitabchi $A E$, Umpierrez GE, Murphy MB, Barrett EJ, Kreisberg RA, Malone JI, et al. Management of hyperglycemic crises in patients with diabetes. Diabetes Care 2001;24(1):131-53.

63. Unger $\mathrm{RH}$, Foster DW. Diabetes Mellitus. In: Wilson JD, Foster DW, Kronenberg HM, Larsen PR (ed). Williams Textbook of Endocrinology. $9^{\text {th }}$ ed. Philadelphia: Saunders, 1998. pp. 1010-2.

64. Fisken RA. Severe diabetic ketoacidosis: the need for large doses of insulin. Diabet Med 1999;16(4):347-50.

65. Chiasson JL, Ris-Jilwan N, Belanger R, Bertrand S, Beauregard $\mathrm{H}$, Ekoe JM, et al. Diagnosis and treatment of diabetic ketoacidosis and the hyperglycemic hyperosmolar state. CMAJ 2003;168(7):859-66.

66. Trachtenbarg DE. Diabetic ketoacidosis. Am Fam Physician 2005;71(9):1705-14.

67. Kitabchi AE, Fisher JN, Murphy MB, Rumbak MJ. Diabetic ketoacidosis and the hyperglycemic hyperosmolar nonketotic state. In: Kahn CR, Weir GC (ed). Joslin's Diabetes Mellitus. $13^{\text {th }}$ ed. Philadelphia: Lea \& Febiger, 1994. pp. 738-70.

68. Riley LJ Jr, Cooper M, Narins RG. Alkali therapy of diabetic ketoacidosis: biochemical, physiologic, and clinical perspectives. Diabetes Metab Rev 1989;5(8):627-36. 
69. Soler NG, Bennett MA, Dixon K, FitzGerald MG, Malins JM Potassium balance during treatment of diabetic ketoacidosis with special reference to the use of bicarbonate. Lancet 1972;2(7779):665-7.

70. Morris LR, Murphy MB, Kitabchi AE. Bicarbonate therapy in severe diabetic ketoacidosis. Ann Intern Med 1986;105(6):836-40.

71. Posner JB, Plum F. Spinal-fluid $\mathrm{pH}$ and neurologic symptoms in systemic acidosis. N Engl J Med 1967;277(12):605-13.

72. Thompson $\mathrm{CH}$, Syme PD, Williams EM, Ledingham JG, Radda GK. Effect of bicarbonate administration on skeletal muscle intracellular $\mathrm{pH}$ in the rat: implications for acute administration of bicarbonate in man. Clin Sci (Lond) 1992;82(5):559-64.

73. Ritter JM, Doktor HS, Benjamin N. Paradoxical effect of bicarbonate on cytoplasmic pH. Lancet 1990;335(8700):1243-6.

74. Bellingham AJ, Detter JC, Lenfant $\mathrm{C}$. The role of hemoglobin affinity for oxygen and red-cell 2,3-diphosphoglycerate in the management of diabetic ketoacidosis. Trans Assoc Am Physicians 1970;83:113-20.

75. Matz R. Rationale for not using bicarbonate. N Y State J Med 1976;76(8):1299-303.

76. Wilson HK, Keuer SP, Lea AS, Boyd AE III, Eknoyan G. Phosphate therapy in diabetic ketoacidosis. Arch Intern Med 1982;142(3):517-20.

77. Fisher JN, Kitabchi AE. A randomized study of phosphate therapy in the treatment of diabetic ketoacidosis. J Clin Endocrinol Metab 1983;57(1):177-80.

78. Cefalu WT. Diabetic ketoacidosis. Crit Care Clin 1991; 7(1):89-108.

79. Troy PJ, Clark RP, Kakarala SG, Burns J, Silverman IE, Shore E. Cerebral edema during treatment of diabetic ketoacidosis in an adult with new onset diabetes. Neurocrit Care 2005;2(1):55-8.
80. Glaser NS, Wootton-Gorges SL, Marcin JP, Buonocore MH, Dicarlo J, Neely EK, et al. Mechanism of cerebral edema in children with diabetic ketoacidosis. J Pediatr 2004; 145(2):164-71.

81. Lam TI, Anderson SE, Glaser N, O'Donnell ME. Bumetanide reduces cerebral edema formation in rats with diabetic ketoacidosis. Diabetes 2005;54(2):510-6.

82. Isales CM, Min L, Hoffman WH. Acetoacetate and betahydroxybutyrate differentially regulate endothelin-1 and vascular endothelial growth factor in mouse brain microvascular endothelial cells. J Diabetes Complications 1999; 13(2):91-7.

83. Lottenberg SA, Hidal JT, Neto MC. Cetoacidose Diabética e Hipoglicemia. In: Knobell E (ed). Condutas no paciente grave. 2a ed. Rio de Janeiro: Atheneu, 1998. pp. 491-8.

84. Melo MS, de Lolio CA, Lucena MA, Kirzner CF, Martins SM, Barros MN. Causas múltiplas de morte em diabéticos no Município de Recife, 1987. Rev Saude Publica 1991; 25(6):435-42.

85. Franco LJ, Mameri C, Pagliaro H, lochida LC, Goldenberg P. [Diabetes as primary or associated cause of death in the state of Sao Paulo, Brazil, 1992]. Rev Saude Publica 1998; 32(3):237-45.

86. de Lima AK, Schmidt MI, Duncan BB. Mortalidade por complicações agudas do Diabetes Mellitus: um bom indicador de saúde para o Brasil? Cad de Saúde Pública 2006; (in press).

Endereço para correspondência:

Bianca Barone

Rua Senador Muniz Freire 50/504

20541-040 Rio de Janeiro, RJ 